\title{
On the Maximum Modulus of Entire Functions and Their Derivatives: corrigendum
}

By

\author{
P. K. Kamthan, Kanpur, India \\ (Received July 30, 1968)
}

Introduction: In an earlier paper ([2], Theorem(iii)) we have shown amongst various results the following:

$$
M_{(s)}(r)<\mu(r) r^{(s+1) e-s+\varepsilon} ; s=1,2, \ldots,
$$

for all $r>r_{0}$ (for notations and definitions see the above mentioned paper).

D. V. V. Wend [Math. Reviews, Vol. 33, 1454, (1967)] has noted an error in the proof of this result, although the result is correct - an error in the summation of an infinite series occuring in the proof. Although this error can be rectified, yet we wish to give a completely different proof of (1) depending on a well-known result of VALIRON.

Proof of (1): Let $\mu_{(s)}(r)$ and $\nu_{s}(r)$ correspond to the $s$-th derivative of $f(z)$ in the usual way (we follow the definition of $\mu_{(s)}(r)$ given in [1]). Then following ValiRoN ([4], p. 32), one has:

$$
M_{(s)}(r)<\mu_{(s)}(r)\left[2 v_{s}\left(r+r / v_{s}(r)\right)+1\right] .
$$

However, to a given $\varepsilon>0$ ( $\varepsilon$ is not necessarily the same at every step)

$$
v_{s}(r)<r^{0+\varepsilon} \text { for all } r \geq r_{0}=r_{0}(\varepsilon) .
$$

Therefore, denoting by $K$ various constants $(>0)$,

$$
M_{(s)}(r)<K \mu_{(s)}(r) r^{e+\varepsilon}, \text { for all } r \geq r_{0}(\varepsilon) ;
$$

also ([3], Theorem 2)

$$
\mu_{(s)}(r) \leq K \mu(r)\left\{v_{s}(r) / r\right\}^{s},
$$

and so using (2) again, 


$$
M_{(s)}(r)<K \mu(r) r^{o s+\varrho-s+\varepsilon}, \text { for all } r \geq r_{0}
$$

and this leads to (1).

\section{References}

[1] KaMthav, P. K.: On the maximum term of an integral function and its derivatives (II). Mh. Math. 68, 125-129 (1964).

[2] KaMthaN, P. K.: On the maximum modulus of entire functions and their derivatives. Mh. Math. 69, 407-409 (1965).

[3] KaMthav, P. K. and P. C. Dash: On the derivatives of an entire function. Bull. Acad. Polonaise Sci., 16, 387-392 (1968).

[4] Vafiron, G.: Integral Functions. New York: Chelsea Publications, 1949.

University of Waterloo, Waterloo, Canada

and

Indian Institute of Technology, Kanpur, India 